\title{
2-d Robotic Arm Control using EMG Signal
}

\author{
Aditya Veer Singh Rana \\ Centre for Development of \\ Advanced Computing, Mohali, \\ Punjab, India
}

\author{
Ridhi Aggarwal \\ Centre for Development of \\ Advanced Computing, Mohali, \\ Punjab, India
}

\begin{abstract}
In the presented work, a robotic arm is controlled using the EMG signal acquired from the electrodes attached to the human arm. The EMG signals are acquired from three different muscle groups of the upper forearm. The EMG signal is very noisy and of the order of micro volts. Faithful extraction of the EMG signal is the prime objective in the presented work. The acquired signal is then fed to signal conditioning unit consisting of signal acquisition, amplification, rectification and final filtration. The output of the signal conditioning unit is then converted to digital signal using a 16-bit serial ADC. The digitized signal is used as feedback and control signal for final control of the robotic arm. The robotic arm is attached to the shaft of the stepper motor for motion. Stepper motor is interfaced using microcontroller. There may be more than one stepper motor to give multi-dimensional motion to robotic arm. It can be assumed that with one single stepper motor, ideally we can have $360 / \theta$ no. of positions ( $\theta$ being the step angle). Presently we have used only two stepper motors for up-down motion at two joints thereby enabling to give $(360 / \theta) 2$ no. of positions.
\end{abstract}

\section{General Terms}

Robotic Arm, Acquisition, EMG, Signal Acquisition.

\section{Keywords}

$\mathrm{SCU} \rightarrow$ Signal Conditioning Unit, ADC $\rightarrow$ Analog to Digital Converter, EMG $\rightarrow$ Electromyography, Amplification, Degrees of Freedom, Stepper Motors, Control Algorithm.

\section{INTRODUCTION}

Electromyography referred to as measuring muscle activation via electric potential. This paper presents the non-invasive method of measuring electromyogram signal from the human forearm muscle and then digitized signal is used as feedback and control signal for final control of the robotic arm. The research has been conducted on the control of the multifunctional prosthetic arm through electromyography (EMG) signals from the muscles of forearm. The research is inspired by the previous work study in the area of usage of EMG signals for pattern classification [1]. The research at CDAC, Mohali involves several issues, such as real-time recording of EMG signals, performing feature extraction and pattern classification, stepper motor-control of hand actuators and interfacing the computer with the external devices. The success of feature extraction depends on the quality of measured EMG signals. As the pattern classification very much depends on the effectiveness of the feature extraction. In other words the EMG signal acquisition is a precondition for feature extraction. The main objective of this paper is to design and develop hardware and software for measuring EMG signals from human forearm muscles in real time and extract it to guide and control the robotic arm. Thus developed sensory/control system would then serve as a test bed for the future development of the multifunctional prosthetic arm, also called "The new generation prosthetic arm".

\section{EMG SIGNAL}

The EMG signal is a small electrical current that is generated when a muscle is contracted. Since the EMG signal levels are too low to be directly captured by the computer, it is required to amplify the signal to a TTL level (which ranges between -5 volts and +5 volts). With the properly amplified EMG signal, the computer can read and store the data in a file format or to use EMG readings in real time. The amplifier that is employed in the reading and recording of EMG signals must be specifically designed to read biological electrical signals. Consequently, many critical factors must be considered. For example, noise and artifact problems could distort the signal. Additional DC current could also add offset to the EMG signal. Providing an adequate ground reference is also an important problem. Finally, because the equipment will be attached to the forearm during the EMG recording, the size of the device should also be taken into consideration.

The measurement of the electrical activity of the muscle is the result of a signal generated in the brain which is transmitted through the nervous system to the motor neuron attached to the muscle fibers in the muscle. The motor neurons resulting in a depolarization/repolarization wave throughout the muscle fiber. This wave creates an action potential in the muscle fibers resulting in the movement of electrical charges. This electrical activity produces an electrical signal in the muscle which can be picked up by well-placed electrodes on the surface of the skin. The acquired signal is EMG signal [2]. This new signal consists of a series of spikes whose amplitude depends on the amount of force delivered by the biceps-the stronger the contraction of the muscle, the larger the amplitude of the EMG signal. The frequency of the spikes is the firing rate of the motor neurons. Since the amplitude of the EMG signal is directly related to the force exerted by the muscle, it is used to determine the force signal sent to the robotic arm.

\section{SIGNAL CONDITIONING UNIT}

Muscle tissues conduct electrical potential similar to the way nerves do and these electrical impulses are called action potentials. The information present in the muscle action potential can be recorded by applying the surface EMG method. It is important to consider two main issues when the detection and recording of EMG signals occur. The first is the signal-to-noise ratio. It is the ratio of energy in the EMG 
signals to the energy in the noise signal. Noise is something that is not desired in a pure EMG signal. The second one is the distortion of the signal. The EMG signal is picked up by electrode and then amplified. It usually needs thousand times of amplification before it could be shown on a display and can be recorded. The point of interest of the signal is the amplitude, which has a range between 0 to 20 millivolts (peak to peak). The frequency of an EMG signal is between 0 to 500 Hz. However, the usable energy of EMG signal is between 50 to $150 \mathrm{~Hz}$. Many dependent factors could affect surface EMG. Thus to avoid the distortion of the signal a signal conditioning unit is incorporated by utilizing an amplifier circuit, Multiplexer, Filter and a buffer amplifier.

Five steps include in the development of signal conditioning unit:

\subsection{Electrode Positioning}

For electrode placement four muscles are involved:

a) Flexor Carpi Ulnaris-wrist flexion with ulnar deviation.

b) Palmaris longus-assist in wrist flexion.

c) Extensor Carpi Radialis-assist in extension and radial abduction of wrist.

d) Extensor digitorum-extension of four fingers and aids in extension of wrist (forward/backward).

For this placement body surface electrodes are used and are placed in the respective muscles for the extraction of the EMG signal [3]. Figure 1 showing the proper positioning of electrodes. There are various factors such as electrodes shape, sizes, positions, orientations and inter electrode distance which are responsible for surface EMG recording. [4] For the EMG electrodes it is necessary to have proper contact with the skin so that a stable transition can be achieved with low noise signal. Being in low impedance we have used $\mathrm{Ag} / \mathrm{AgCl}$ electrodes which are pre-gelled electrodes to reduce electrodeskin impedance. To avoid destabilization of the electric layer and difference in impedances inter electrode distance is fixed using elastic bands and tapes.

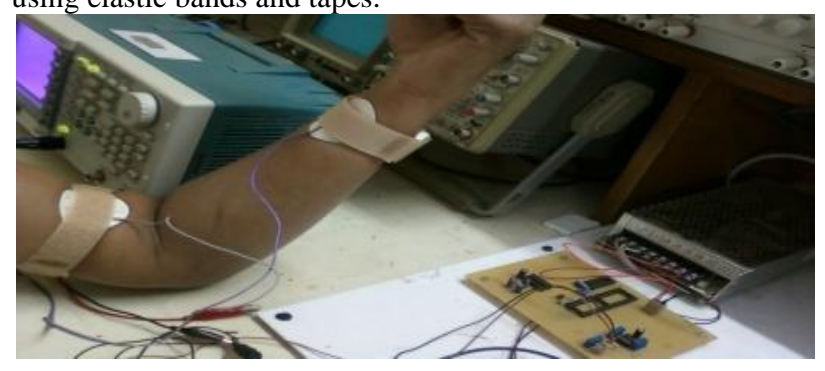

Fig 1: Electrode Placement

\subsection{Signal Acquisition}

The signal acquisition phase is used to measure body's nervous system's electrical impulses to activate muscle fibers. Signal acquired by the electrodes are fed into an operational differential amplifier via wires to boost the level of the input signal to match the requirements of the instrumentation system or to match the range of the analog-todigital convertor, thus increasing the resolution and sensitivity of the measurement. Differential operational amplifier INA106 with precision gain of 10 is used for the amplification which have high input impedance and high CMRR to neglect the unwanted signals [5].

\subsection{Signal Conditioning-Amplification}

The signal acquisition phase measures very small signals containing low frequency noise. Thus, the output of INA106 is then fed to the operational amplifier TL072 [6]. The signal conditioning phase amplify the signal and also inverts it to fix the required gain. A capacitor is incorporated to remove DC error offset in a signal.

\subsection{Signal Conditioning-Rectification}

This phase is used to rectify the signal (using an active fullwave rectifier). The rectifier takes the negative portion of the signal and turns it positive so the entire signal falls within the positive voltage region. Operational amplifier TL072 is used with two zener diodes for rectifying the signal to settle the suitable level.

\subsection{Signal Conditioning-Final Signal}

The analog signal is obtained by ADC unit and a digital signal is passed to microcontroller which requires constant 5 volts supply for its operation to control motors. Thus, readying the final signal and passing it before to ADC unit, an active low pass filter is used to smooth the signal.

\subsection{Signal Processing-ADC-LTC-1867}

The microcontroller P89V51RD2 uses TTL standard level analog interfaces ( +5 volt and -5 volts). An amplification of the signal is needed to boost the signal up to a required level. LTC-1867 [7] which is a 16-bit Analog to Digital converter and its signal-to-noise ratio is typically $89 \mathrm{~dB}$ and is used to provide rectified signals to the computer on chip to further use the feedback and control signal for final control of robotic arm. The shaft of the robotic arm is attached to the robotic arm to obtain its multi-dimensional motion.

\section{ARM CONTROL ALGORITHM}

In the presented work, it is planned to design a robotic arm with two joints control. Therefore, we can have maximum positions computed as:

Say Step Angle, $\theta=7.50$. No. of position availed by one stepper Motor $=360 / 7.5=48$.

Therefore, with two stepper motors at two joints, we can have $2304(=48 \times 48)$ possible locations where the robotic arm can have access ideally. However, some positions are not accessible like in very close vicinity of the lower joint.

\section{POSITION COMPUTATION}

No. of positions of the robotic arm can be illustrated by using the robot arm free body diagram (FBD). The DenavitHartenberg (DH) Convention is the accepted method of drawing robot arms in FBD's [8]. There are only two motions a joint could make: translate and rotate. There are only three axes this could happen on: $\mathrm{x}, \mathrm{y}$, and $\mathrm{z}$ (out of plane). One of a robotic arm functions is to move to a specified location or along a predetermined path so it can perform a task [9]. In the proposed work, motion is consist of the robot itself moving, or of an articulated arm being actuated from a fixed pivot position. Considering the problem of controlling the motion of a very simple articulated arm, a two-segment arm that can move only in the $x-y$ plane and pivots about the position $x=0$, $y=0$. A stepper motor at $(0,0)$ is attached to the first arm segment and controls its angle with respect to the $\mathrm{x}$-axis. A second stepper motor is attached to a second arm segment and 
controls its angle with respect to the $\mathrm{x}$-axis. The computation is based on joint rotation, length of each link and their weight, distance between each stepper motor as the length of the linkage is critical to the mechanical design and computation of positions.

\section{DISTANCE IN ANY DIRECTION}

Considering the two degree of freedom of two respective joints the maximum distance in any direction is proportional to the joint angles of the robotic arm controlled by stepper motor of two joints. Due to the nonlinearity of the model equations and the large number of unknown parameters per muscle that makes the overall analysis rather difficult. Similar studies [10] that used musculoskeletal models, focusing on a limited number of muscles and actuated Degree of Freedoms. The distance in any direction can be achieved by the kinematics of manipulators which assists the geometrical and time-based properties of the motion. Manipulators with their links allow relative motion of neighboring links which are connected by joints to the stepper motor. These joints are usually instrumented to allow the relative position of neighboring links to be measured and the number of independent position variables that would have to be specified in order to locate all distance in respective directions.

\section{STEPPER MOTOR INTERFACE}

The stepper motors used in this were 6 pin unipolar ones. Two pins are connected to the $12 \mathrm{~V} \mathrm{DC}$ power supply and the other four pins are connected to the microcontroller. Figure 2 shows the block diagram for the control of robotic arm using the P89V51RD2 microcontroller. The other four pins belonged to the two coils of the stepper motor. That is each coil contains two wires. Two wires of the same coil should not be given the same pulse. That is if wire 1a of coil is given high pulse, $1 \mathrm{~b}$ wire should be given low pulse and vice versa. The order in which the pulses are given to the wires determines the direction of rotation of the motor shaft. ULN 2803 is used to drive the stepper motor from the microcontroller. The COM pin number 10 is connected to 12 volt supply for driving motors which have back emf in coils. Programming for the stepper motors was done in Keil $\mu$ vision-4 software. Coding in P89V51RD2 is done in C format and it was burnt to the microcontroller using the Flash Magic programmer from NXP. The Flash Magic programmer was connected with the computer and the program was loaded into it using the Flash Magic software. Then the program is stored into the P89V51RD2 microcontroller using the flash burner. Port D is connected to one stepper motor and Port $\mathrm{C}$ is connected to another one. $12 \mathrm{~V} \mathrm{DC}$ power supply is given to both the development board and the two stepper motors through a $12 \mathrm{~V}$ DC adaptor. Later, the hardware parts are built and are connected together and programming is done and loaded into the microcontroller. Finally, power supply is given and the system is operated. Figure 3 showing block diagram for stepper motor interfacing.

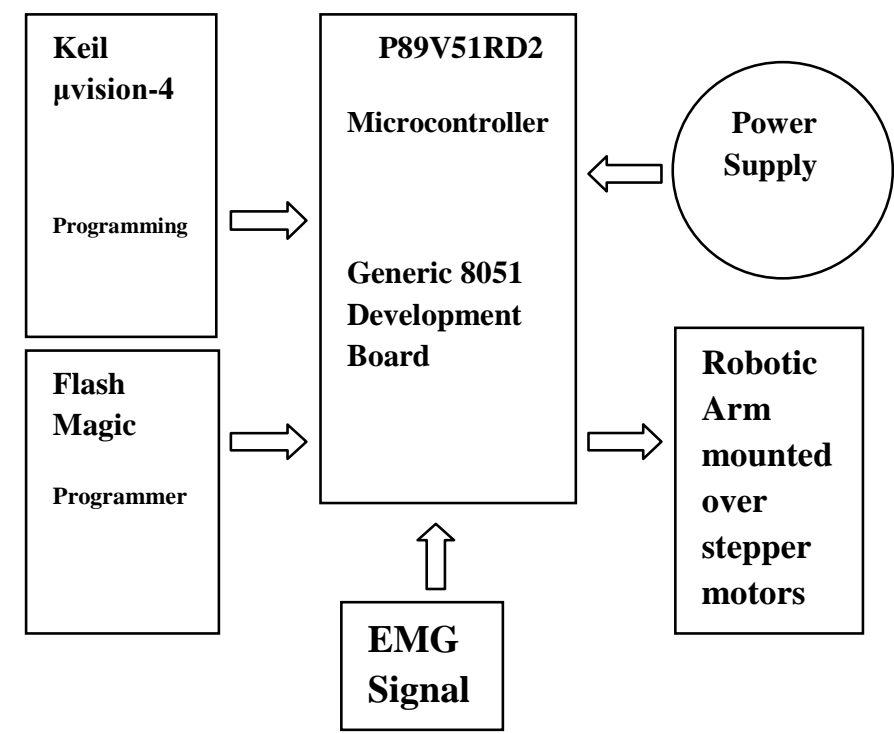

Fig 2: Control of Robotic Arm

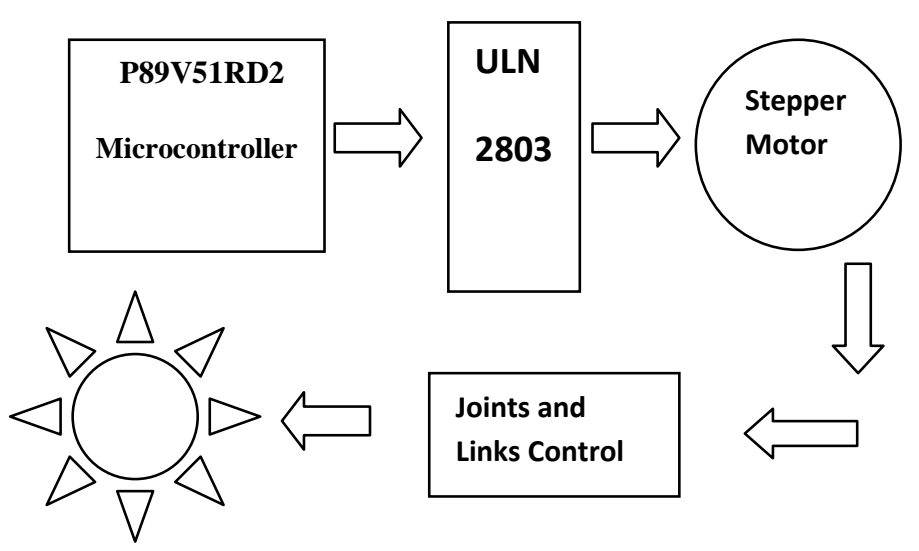

Fig 3: Block Diagram of Stepper Motor Interfacing

\section{MECHANICAL CONSTRUCTION}

For the construction of robotic arm nylon rods of thickness $1 \mathrm{~cm}$ are used. The length of the rod links is critical to the mechanical design. If the links are too long, the stepper motors due to not having enough torque cannot lift the load. If the rod links are short, it will reduce the arm reach and motors might not move. Thus, rods are cut according to the required shape and dimensions. The construction also maintains low costing parameters for the final assembly in the mechanical design. The joints and links are made by installing using stepper motors and gears handling a combined torque of 12 $\mathrm{kg}-\mathrm{cm}$ to lift the entire arm. The motors capacity to lift the loads is specified in terms of torque

(kg-cm/N-m/oz-in) 
The torque equation is specified as follows:

Torque $=$ Force $\times$ Distance $\times \operatorname{Sin} \Theta$

Where, ' $\theta$ ' represents the angle between the 'Force' acting on the shaft and 'Distance' represent how far the force is applied from the centre of the shaft.

\section{RESULTS}

The EMG signal received at microcontroller is a 16-bit ADC ranging from 0 - 65535. However, multi thresholds $\{\mathrm{T} 1, \mathrm{~T} 2$, $\mathrm{T} 3, \ldots \mathrm{Tn}$ \} can be selected in order to control/activate the robotic arm at different positions and for different states/movement like MOVE, STOP, LEFT, RIGHT, UP and DOWN. The positions are determined using the two joints degree of freedom shown in Table 1.

\begin{tabular}{|l|l|l|}
\hline Threshold & T1 $<00$ & MOTOR STOP \\
\hline Threshold & $501<\mathrm{T} 2<1000$ & MOVE \\
\hline Threshold & $1001<\mathrm{T} 3<1500$ & MOVE LEFT \\
\hline Threshold & $1501<\mathrm{T} 3<2000$ & MOVE RIGHT \\
\hline Threshold & $2001<\mathrm{T} 3<2500$ & MOVE UP \\
\hline Threshold & $2501<\mathrm{T} 3<3000$ & MOVE DOWN \\
\hline
\end{tabular}

Table 1. Robotic arm at different positions.

The motion is intended to obtain the different states and number of positions is either using stepper motors as well as using servo motors. The arm may be controlled using the above given thresholds and can be extended for any application.

\subsection{Experimentation Results}

The extraction of EMG signal is processed to operate the 2dimensional control of robotic arm and obtaining 3 Degree's of Freedom. Some results obtained on CRO showing the output signals at different phases of signal acquisition are given below:

\section{Signal Acquisition}

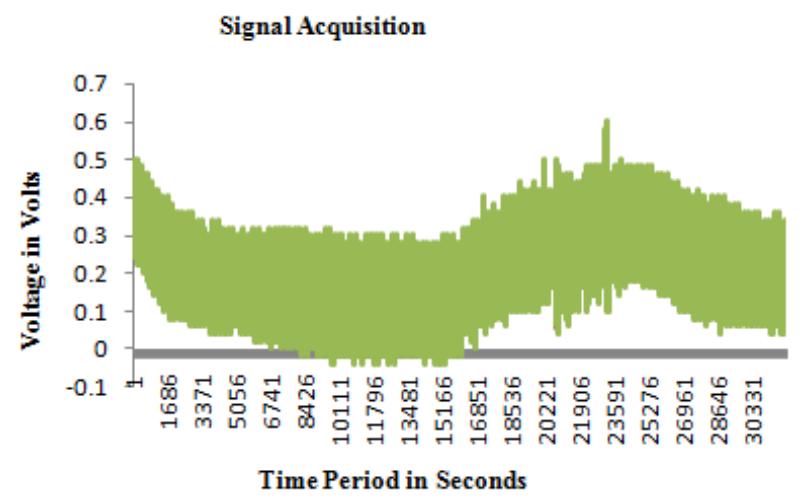

\section{Signal Acquisition-Conditioning}

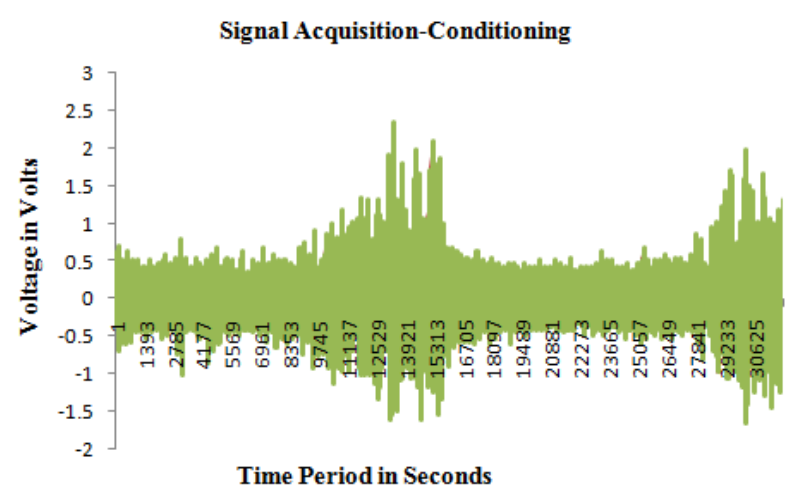

\section{Signal Acquisition-Rectification}

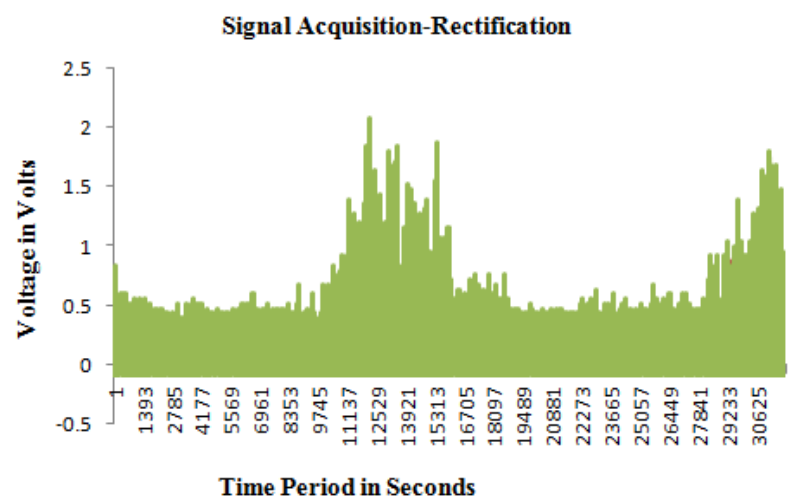

Signal Acquisition-Final Signal

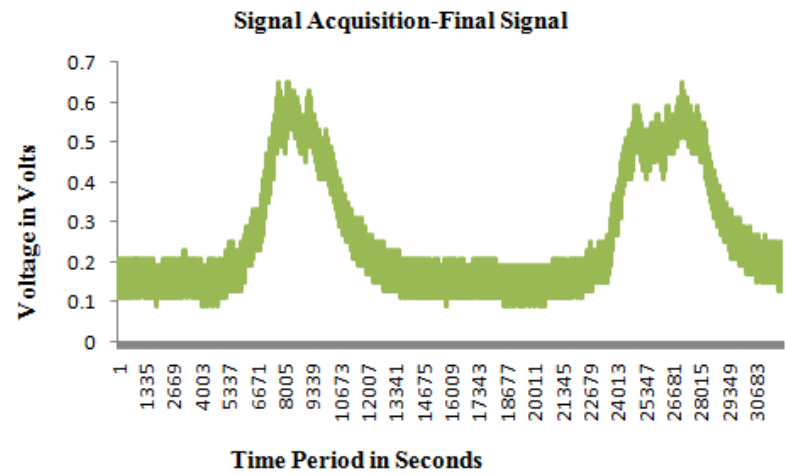




\section{CONCLUSION}

The presented work is based on the faithful extraction of EMG signals from human body. The EMG signal acquired is maintained under the range of 0 to 5 volts and can be accessible by any ADC unit. The digital data obtained after conversion is utilized to read by microcontroller unit. The data received from the microcontroller port is further tested on various class of motors like stepper motors, servo motors and DC motors resulting in a mechanical model which is designed to organize the robotic arms versatility with respect to number of position and rotation in various applications. The threshold selection is a careful task and depends upon the end application. The thresholds given above are general one and may not suit to every application. But a model may be developed for any particular application like typing, lifting parts or any repetitive tasks. The scope of the design can further be advanced in the area of arm prosthesis, rehabilitation engineering, diagnosis in the medical and sports science.

\section{ACKNOWLEDGEMENTS}

We thank Centre for Development of Advanced Computing, Mohali and our guide Mr. Vikas Goel- Sr. Project Manager at C-DAC, Mohali for the motivation and encouragement to make this research work as successful one.

\section{REFERENCES}

[1] Artemiadis, P.K, "EMG-Based Control of Robot Arm using Low-Dimensional Embeddings", IEEE Transactions on Robotics. Vol. 26, Issue: 2, pp. 393- 398, 2010.

[2] F. E. Zajac, "Muscle and tendon: Properties, models, scaling, and Application to biomechanics and motor control," in Proc. Crit. Rev. Biomed. Eng., vol. 17, Boca Raton, FL: $\quad$ CRC, 1989, pp. 359-411.
[3] d'Avella , A. Portone, L. Fernandez and F. Lacquaniti "Control of fast-reaching movements by muscle synergy combinations", J Neurosci., vol. 25, no. 30, pp. 7791-7810, 2006.

[4] Hermens HJ, Freriks B, Merletti R, et al. Recommendations for surface electromyography SENIAM 8: Roessingh Research and Development. Enschede. 1999.

[5] Differential operational amplifier INA106, datasheet.

[6] Operational amplifier TL072, datasheet.

[7] ADC LTC-1867, datasheet

[8] Thomas, U, Maciuszek, I.; Wahl, F.M. "A Unified Notation for serial, parallel, and hybrid Kinematic Structures", IEEE Proceedings of Robotics and Automation. Vol. 3, pp. 2868- 2873, 2002.

[9] Society of Robots tutorials.

[10] Parasuraman, S. "An EMG-driven Musculoskeletal model for robot assisted stroke Rehabilitation system using sliding mode control". Mechatronics and its Applications (ISMA), $7^{\text {th }}$ pp.1-6,2010.

\section{AUTHORS' PROFILE}

${ }^{1}$ Aditya Veer Singh Rana has received B.Tech. degree in Electronics \& Instrumentation from C.C.S University, Meerut in 2010 and is pursuing M.Tech. thesis work in Embedded Systems Technology from C-DAC, Mohali. His expertise field of interest is embedded system design and control technologies.

${ }^{2}$ Ridhi Aggarwal is pursuing M.Tech. thesis work in Electronics Product Design \& Technology from C-DAC, Mohali. Her expertise field of interest is Electronics product design and control technologies. 